\title{
Análisis de decisión: coste-efectividad en el tratamiento quirúrgico de la neuralgia del trigémino
}

\author{
I. Mora Díaz*, J. E. Martínez Suárez**, L. S. Fernández Hernández***, A. Rosa Gutiérrez*, \\ R. E. Jiménez Paneque* y T. Hidalgo Costa*
}

Departamento de Investigaciones y Proyectos. Hospital Clínico Quirúrgico Hermanos Ameijeiras. La Habana, Cuba.

Mora Díaz I, Martínez Suárez JE, Fernández Hernández LS, Rosa Gutiérrez A, Jiménez Paneque RE, Hidalgo Costa T. Análisis de decisión: coste-efectividad en el tratamiento quirúrgico de la neuralgia del trigémino. Rev Soc Esp Dolor 2013; 20(4): 161-169.

\begin{abstract}
Introduction: Trigeminal neuralgia is a facial pain syndrome known and characterized by severe, intermittent, electric shocklike, shooting pain in the face for which a number of surgical therapies have been used. To date no ideal, minimally invasive, patient-acceptable treatment is available, hence both patients and specialists face substantial uncertainty when making decisions regarding therapeutic action.

Objective: To identify which surgical treatment for TN offers the best opportunity in terms of cost-effectiveness.

Material and method: A study was performed using a decision analysis/cost-effectiveness analysis design considering five surgical alternatives in the management of $\mathrm{TN}$ : radiofrequency thermocoagulation, glycerol microcompression, balloon microcompression, radiosurgery, and microvascular decompression. Data were obtained from the literature. A review was carried out of the MEDLINE database from 2000 to 2010 via PubMed Central. Studies were selected that addressed primary trigeminal neuralgia and demonstrated pain relief and reduced mortality and complication rates. In all 196 studies were identified but only 22 (11.22\%) were eligible for the study. The software package used
\end{abstract}

Financiación: Ninguna

Conflicto de intereses: Todos los autores están de acuerdo con las normas de publicación.

Recibido: 02-10-12.

Aceptado: 05-12-12 was the DATA 3.5 for Health Care, version 3.5.5, program by TreeAge Software Inc.

Results: Microvascular decompression and thermocoagulation are the techniques more likely to provide effective pain relief, with expected values of 0.8946 and 0.8863 , respectively. For the relationship between cost and outcome pain-free days were considered; the best choice in terms of cost-effectiveness is surgery and thermocoagulation, with a lower value in the marginal cost-effectiveness ratio: $\$ 171.58$ for an effectiveness of $89 \%$ per treated patient.

Conclusion: Considering that both radiofrequency thermocoagulation and microvascular decompression are the most commonly used surgical procedures because of their high effectiveness, we conclude that radiofrequency thermocoagulation is more beneficial, has few complications, and is less costly.

Keywords: Trigeminal neuralgia management. Cost-effectiveness.

\section{RESUMEN}

Introducción: La neuralgia trigeminal es un síndrome de dolor facial conocido y caracterizado por dolores severos, intermitentes, eléctricos y como de sacudidas en la cara, para el cual han sido aplicados diversos tratamientos quirúrgicos, hasta la fecha, no hay un tratamiento ideal que sea invasivo en grado mínimo y aceptable para el paciente, como consecuencia, los pacientes y los especialistas se enfrentan a una incertidumbre considerable al hacer decisiones sobre la conducta terapéutica.

Objetivo: Identificar cuál de los tratamientos quirúrgicos para la NT brinda la mejor oportunidad en términos de coste-efectividad.

Material y método: Se realizó un estudio con un diseño de Análisis de Decisión: coste-efectividad considerando cinco alternativas quirúrgicas en el tratamiento de la NT: termocoagulación por radiofrecuencia, microcompresión con glicerol, microcompresión con balón, radiocirugía, y microdescompresión vascular. Los datos se obtuvieron de la literatura. Se revisó la base de datos MEDLINE desde el año 2000 hasta el 2010, a través de PubMed Central. Se seleccionaron los estudios que abordaran la neuralgia trigeminal primaria y mostraran resultados relacionados con el alivio del dolor, la tasa de mortalidad o 
la presencia de complicaciones. Se identificaron 196 estudios pero solo 22 (11,22 \%) fueron elegibles para el estudio. Se utilizo el programa DATA 3.5 for Health Care, versión 3.5.5 de TreeAge Software Inc.

Resultados: La microdescompresión vascular y la termocoagulación son las técnicas que ofrecen mayores probabilidades de efectividad para el alivio del dolor con valores esperados de 0,8946 y 0,8863 . Para la relación que se establece entre coste y resultado se consideró los días libres de dolor. La mejor elección costeefectividad es el tratamiento quirúrgico con termocoagulación con menos valor en la razón coste-efectividad marginal, \$171.58 para una efectividad de la cirugía de $89 \%$ por cada paciente tratado.

Conclusión: Considerando que la termocoagulación por radiofrecuencia y la microdescompresión vascular constituyen las variantes quirúrgicas más utilizadas por la alta efectividad, concluimos que la termocoagulación por radiofrecuencia es más beneficiosa, ofrece escasas complicaciones y es menos costosa.

Palabras claves: Tratamiento neuralgia trigeminal. Costeefectividad.

\section{INTRODUCCIÓN}

La neuralgia trigeminal (NT) es un síndrome de dolor facial conocido y caracterizado por dolores severos, intermitentes, eléctricos y como de sacudidas en la cara. La ansiedad y la depresión son consecuencias comunes de estos síntomas, que además originan un deterioro en la calidad de vida (1). El tratamiento medicamentoso más conocido para esta dolencia son los anticonvulsivos siendo la carbamacepina el fármaco de primera elección; pero solo es eficaz en el $25 \%$ de los pacientes y alrededor de un $8 \%$ desarrollan intolerancia a la droga. De modo que la cirugía se convierte en el tratamiento de elección de estos pacientes (2-4).

Según la literatura revisada, desde la primera publicación científica sobre NT por John Fothergill en 1773, han sido aplicados diversos tratamientos quirúrgicos y muchos cirujanos han apoyado con datos la superioridad de su técnica quirúrgica $(1-4,5)$. Sin embargo, hasta la fecha, no existe tratamiento quirúrgico ideal que sea invasivo en grado mínimo y aceptable a la vez para el paciente, es decir, que alivie el dolor inmediata y completamente, carezca de complicaciones y no tenga efectos secundarios ni fracasos o recurrencias.

Dado que la NT no es una condición que amenaza la vida, la decisión para realizar una intervención quirúrgica no es una cuestión de vida o muerte en el sentido convencional. No obstante, el dolor y su efecto en la calidad de vida es inquietante y los tratamientos pueden ser costosos, por tanto, los pacientes y los especialistas se enfrentan a una incertidumbre considerable al tomar decisiones sobre la conducta terapéutica de un paciente con NT.

Existe una gran variedad de tratamientos quirúrgicos disponibles que se encuentran a menudo en conflicto debi- do a los propios resultados. Algunos procedimientos, como la cirugía por microdescompresión vascular ha demostrado eficacia en un porcentajes alto de pacientes, pero se acompañan de un riesgo elevado de complicaciones por la intervención (6-9). En otros como la radiocirugía las complicaciones son menos, pero la eficacia disminuye (1013). Por consiguiente, es difícil aconsejar a pacientes sobre cuál es el mejor tratamiento. Las preferencias personales y la experiencia, las actitudes hacia el riesgo en nombre del paciente y el cirujano, y factores clínicos (como la edad del paciente y su estado general de salud) muchas veces permiten al médico sugerir algún procedimiento en concreto.

El análisis de decisión en el contexto de los cuidados o intervenciones sobre la salud constituye una herramienta eficaz para ayudar a determinar el tratamiento que se erija como el mejor en base a un balance entre riegos y beneficios junto a la satisfacción de las perspectivas del paciente $(14,15)$.

A medida que la toma de decisiones en salud se ha convertido en un proceso cada vez más complejo, la mejor evidencia disponible y las medidas adecuadamente estructuradas han ido reemplazando la opinión de los expertos. $\mathrm{El}$ análisis de decisión por su naturaleza permite incluir elementos cuantitativos que reflejan la mejor evidencia disponible $(16,17)$.

El presente trabajo se encamina a identificar cuál de los tratamientos quirúrgicos aceptados hoy para la NT brinda al paciente la mejor oportunidad en términos de costeefectividad.

\section{OBJETIVO}

Identificar cuál de las estrategias quirúrgicas en el tratamiento de la neuralgia trigeminal produce una mayor utilidad en términos de coste-efectividad.

\section{MATERIAL Y MÉTODO}

Se decidió emplear un diseño de análisis de decisión: coste-efectividad para abordar el problema, este método utiliza un modelo de árbol de decisión que consiste en un gráfico donde se representan mediante líneas y símbolos los diferentes cursos de acción y sus posibles resultados. Se siguieron los pasos descritos para el análisis de decisión y luego se recopilaron los datos necesarios para el análisis de coste-efectividad.

- Construcción del árbol de decisión. Para esta tarea se reunió un grupo de expertos que señalaron los cursos de acción. Se consideró que para cada alternativa terapéutica se podían señalar 4 cursos: 1) la muerte, 2) la aparición de complicaciones graves que no resultaran en fallecimiento, 3) la aparición de complicaciones no 
graves y 4) la no aparición de complicación alguna. El curso posterior para las ramas del árbol que no eran muertes consideraba la posibilidad de que el paciente quedara sin dolor alguno o con dolor. El final del árbol se cuantificó con la efectividad que se definió como el número de pacientes del total que quedaba libre de dolor (expresado en proporciones).

- Obtención de la información. La principal fuente de datos fue una revisión bibliográfica de la literatura con información referente al tratamiento quirúrgico de la NT, fue revisada la base de datos MEDLINE desde el año 2000 hasta el 2010, a través de PubMed Central. Se seleccionaron, como candidatos a ofrecer datos, los estudios que abordaran la neuralgia trigeminal primaria y mostraran resultados relacionados con el alivio del dolor, la tasa de mortalidad o la presencia de complicaciones. Se identificaron 196 estudios que describieron los resultados de varios procedimientos quirúrgicos para el tratamiento de la NT, pero solo 44 estudios $(22,4 \%)$ se ajustaron con los criterios establecidos y $22(11,22 \%)$ fueron fuentes fidedignas para estimar la probabilidad de quedar libres de dolor para cada técnica quirúrgica.

- Análisis de decisión. Se consideraron cinco alternativas quirúrgicas en el tratamiento de la NT: termocoagulación por radiofrecuencia, microdescompresión con balón-catéter, microdescompresión con glicerol, radiocirugía (técnicas mínimamente invasivas con abordaje percutáneo), y la microdescompresión vascular (técnica invasiva con abordaje intracraneal). El modelo para el análisis siguió al paciente hasta evaluar los resultados inmediatamente después de la cirugía y el resultado final se considero en dos posibilidades: con o sin dolor, como se explicó antes (Fig. 1).

- Asignación de probabilidades. Para cada curso de acción se estimaron las siguientes probabilidades en los nodos de oportunidad: probabilidad de morir por causa de la cirugía, probabilidad de presentar complicaciones graves (se refiere a la probabilidad de presentar: fístula carótida cavernosa, enfermedad cerebro-vascular, meningitis, hematoma del lecho quirúrgico), probabilidad de complicaciones no graves (probabilidad de presentar trastornos de la sensibilidad, alteración del reflejo corneal, queratitis, alteraciones de los pares craneales, debilidad del músculo masetero, hematomas locales) (para la clasificación de las complicaciones en graves y no graves se tuvo en cuenta el criterio de expertos) y probabilidad de quedar sin dolor después de realizada la cirugía.

En cada nodo de oportunidad, se calcularon simples promedios de las distintas probabilidades ofrecidas por las referencias bibliográficas. La utilidad empleada fue el porcentaje de efectividad (considerado alivio completo del dolor sin medicación) de la técnica quirúrgica para cada resultado final definido, excepto en caso de muerte donde el porcentaje de efectividad toma valor cero. El análisis de la literatura permitió obtener varias estimaciones de la probabilidad de cada una de las posibilidades evaluadas en cada nodo, estos rangos fueron usados en el análisis de sensibilidad (Tabla I).

\section{Costes}

Los costes están cuantificados en base al sistema de equivalencia económica que se aplicó (un dólar igual a un peso convertible) y se determinaron en base a: salarios, depreciación de los equipos, consumo de agua, energía, materiales gastable e insumos, como lavandería, esterilización, películas radiográficas, reactivos de laboratorio, medicamentos, catéteres intravenosos, prevé el valor por el pago de agua, luz, teléfono, gas, correo, etc.

- Coste-efectividad: el gasto del paciente depende exclusivamente del tratamiento de su enfermedad (patología), esa es la realidad y es por tanto, lo que tiene que mostrar el sistema de costes. La relación que se establece entre su coste y su resultado se conoce como ecuación coste-efectividad, este resultado se puede medir de diversas formas: para nuestro estudio se considero los días libres de dolor.

- Costes variables o directos: en este caso se definen como aquellos que intervienen directamente en la atención o actividad, siendo elementos de los costes directos los gastos que forman parte de la atención o gastos de personal: médicos/as, tecnólogos/as: laboratorio, enfermeras/os y auxiliares.

Para estimar su valor se consideró el tiempo dedicado a la actividad (ocho horas, medio tiempo, tiempo parcial, etc.) y la remuneración. El coste directo de personal se estimó multiplicando el valor de la remuneración en minutos por el tiempo en minutos dedicado a la prestación o actividad. La información se obtuvo a partir del listado de los trabajadores del área con su respectivo cargo y remuneración total. El coste total es el resultado de la consolidación o sumatorio de todos los costes.

- Análisis estadístico: se utilizó el programa DATA 3.5 for Health Care, versión 3.5.5 de TreeAge Software Inc. Se calcularon los valores esperados de cada rama mediante la multiplicación de las utilidades al final de cada rama por las probabilidades de la rama, para cada nodo de oportunidad (que comprende la $p$ de morir por causa de la intervención, $p$ de presentar complicaciones graves o de presentar complicaciones no graves) se calculó un valor esperado de utilidad hasta alcanzar un valor para cada 


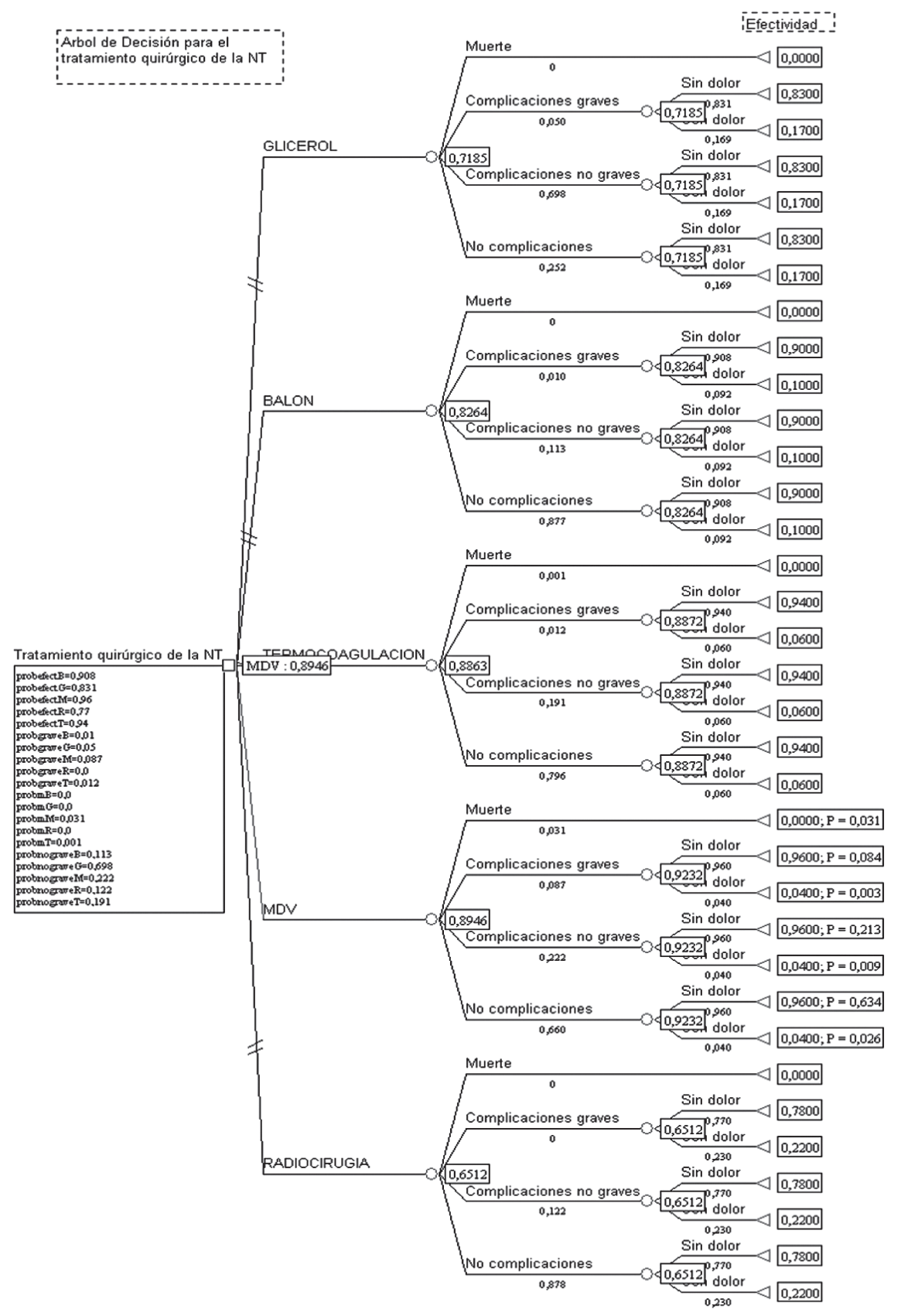

Fig. 1. Árbol de decisión para el tratamiento quirúrgico de la NT.

estrategia de tratamiento quirúrgico. La selección de la mejor alternativa se basó en la mayor utilidad reportada, o sea, la variante quirúrgica que ofrezca una mayor efectividad a los pacientes. Se realizó un análisis de sensibilidad para evaluar si la imprecisión de las estimaciones realizadas afecta a la decisión del análisis. Se consideraron los valores mínimos y máximos de las probabilidades hallados en la literatura. Con los costes estimados para cada técni- ca quirúrgica y sus valores esperados, se calcularon los costes marginales, la efectividad marginal y las razones de coste-efectividad marginales, el escenario analizado consideró el coste de la alternativa y la efectividad ganada, expresándose las mismas en dinero gastado por porcentaje de efectividad ganada con el proceder quirúrgico realizado $(\$ / \%)$. Se consideró como mejor alternativa la de menor valor en la razón coste-efectividad marginal. 
TABLA I. PROBABILIDADES USADAS EN EL ANÁLISIS DE DECISIÓN PARA LA EFECTIVIDAD DE LA CIRUGÍA EN EL TRATAMIENTO DE LA NT

\begin{tabular}{llll}
\hline Resultados & Prob. base & Rango de prob & Fuentes \\
\hline P morir & & & Zakrzewska, 2003 \\
\hline Glicerol & 0 & $0-0,01$ & Scott W,2008 \\
Balón & 0 & $0-0,01$ & Lopez BC,2004 \\
Termocoag & 0,0014 & $0-0,01$ & Editorial,2003 \\
MDV & 0,0312 & $0,001-0,045$ & In Ho Oh et al, 2008 \\
Radiocirug & 0 & $0-0,001$ & Salama H, 2009 \\
\hline P comp graves & & & Silverberg, 1978 \\
\hline Glicerol & 0,05 & $0,0-0,05$ & Adams CB, 1982 \\
Balón & 0,01 & $0,0-0,01$ & Broggi G, 2000 \\
Termocoag & 0,012 & $0,06-0,012$ & Gerald D, 1978 \\
MDV & 0,087 & $0,07-0,09$ & Editorial, 1981 \\
Radiocirug & 0 & 0 & Sengupta R, 1977 \\
\hline P comp no graves & & & Mittal B 1986 \\
\hline Glicerol & 0,698 & $0,01-0,7$ & Bharat M, 1986 \\
Balón & 0,113 & $0,05-0,2$ & Editorial, 1977 \\
Termocoag & 0,191 & $0,05-0,3$ & Barker et al, 1996 \\
MDV & 0,222 & $0,1-0,3$ & Pringle M,1996 \\
Radiocirugía & 0,122 & $0,1-0,3$ & Taha JM, 1997 \\
\hline Éxito & & & Ashkan K, 2004 \\
\hline Glicerol & 0,831 & Ong KS et al. 2003 \\
Balón & 0,908 & $0,70-0,95$ & Tatli M, 2008 \\
Termocoag & 0,931 & $0,89-0,94$ & Spatz, 2006 \\
MDV & 0,966 & $0,90-0,98$ & \\
Radiocirugía & 0,774 & $0,80-0,98$ & \\
\hline & & $0,75-0,90$ & \\
\hline
\end{tabular}

\section{RESULTADOS}

\section{Resultados del análisis de decisión}

Como se muestran en la fig. 1, los valores esperados para los tratamientos fueron:

- Microdescompresión vascular. MDV =0,8946.

- Termocoagulación por radiofrecuencia. Termocoagulación $=0,8863$.

- Microdescompresión con balón-catéter. Balón = 0,8264 .

- Microdescompresión con glicerol. Glicerol = 0,7185.

- Radiocirugía = 0,6512.

La alternativa que ofrece la mayor probabilidad de efectividad para el alivio del dolor es la MDV, con un valor de 0,8946 , seguida de cerca por la termocoagulación.

El análisis de sensibilidad, considerando la $p$ de efectividad para cada técnica quirúrgica, solo encontró variación en la MDV y la termocoagulación, siendo la primera la que más modifica su valor con un rango de 0,0261 (Tabla II).

Para las técnicas que introducen más variación en los valores esperados se realizó un análisis univariado. En las figs. 2 y 3 se observan los valores esperados y las probabilidades de cada alternativa, cuyas utilidades estaban por encima o por debajo del valor del umbral, lo cual demuestra que la decisión fue sensible para algunos cambios en las utilidades introducidas. Para los tratamientos por MDV y termocoagulacion la decisión cambia cuando los valores de la probabilidad de efectividad son menos de 0,951 y 0,949 respectivamente.

\section{Resultados de coste-efectividad}

Las cinco alternativas quirúrgicas fueron evaluadas mediante un simple análisis de coste-efectividad. Las alternativas de tratamiento fueron inicialmente ordenadas por 
TABLA II. ANÁLISIS DE SENSIBILIDAD PARA EL TRATAMIENTO QUIRÚRGICO DE LA NT

\begin{tabular}{llll}
\hline Probabilidades & Rango & $V E$ & Recorrido \\
\hline P efectividad M & $0,90-0,98$ & $0,8863-0,9124$ & 0,0261 \\
\hline P efectividad T & $0,90-0,96$ & $0,8946-0,9039$ & 0,0093 \\
P efectividad R & $0,75-0,90$ & $0,8946-0,8946$ & 0 \\
P efectividad G & $0,70-0,95$ & $0,8946-0,8946$ & 0 \\
P efectividad B & $0,89-0,94$ & $0,8946-0,8946$ & 0 \\
\hline
\end{tabular}

sus costes totales estimados, los costes incrementales y los efectos (\% de efectividad) se calcularon posteriormente.

Como se muestra en la tabla III, el tratamiento con efectos más pequeños que el tratamiento precedente fue eliminado de la comparación, dado que fue dominado por el tratamiento previo. En un segundo paso, después de excluidas las estrategias que fueron dominadas, quedan las alternativas con mejor efectividad, siendo la termocoagulación la que ofrece los mejores resultados coste-efectividad, la razón del coste-efectividad marginal para la termocoagulación y la MDV fue de \$171.58 y \$3684.88 respectivamente, para una efectividad de la cirugía de aproximadamente un $89 \%$ en ambas alternativas. La mejor elección costeefectividad resultó ser el tratamiento quirúrgico con termocoagulación. Los resultados se muestran en un grafico de frontera de eficiencia (Fig. 4).

\section{DISCUSIÓN}

La disyuntiva entre ofrecer la MDV o una técnica mínimamente invasiva se basa en valorar una serie de elementos relacionados con el paciente y su patología. Sin embargo, no siempre se tiene en cuenta el concepto de coste y si contamos con una técnica quirúrgica que fuera menos costosa y que la efectividad superara el $90 \%$, estaríamos en

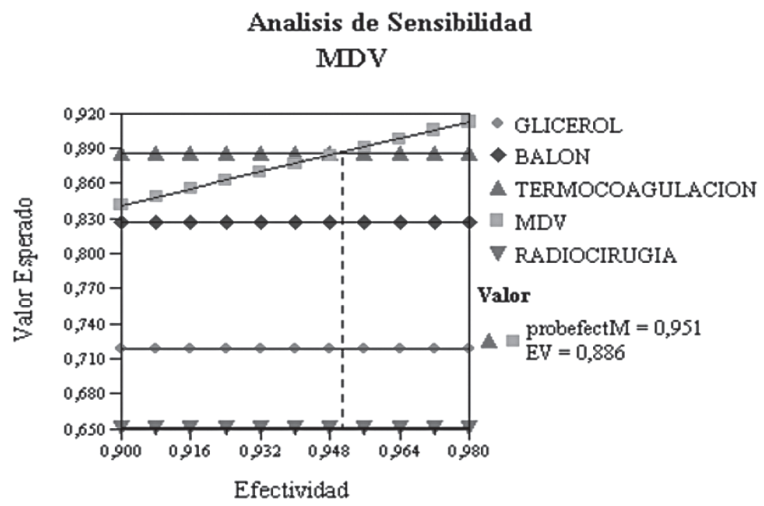

Fig. 2. Análisis univariado de sensibilidad para la MDV. una de las opciones terapéuticas más recomendadas para el tratamiento de la neuralgia trigeminal.

Los procederes mínimamente invasivos tienen ventajas sobre las otras. Las publicaciones de Mittal, Taha y cols. (1822) consideran que la termocoagulación por radiofrecuencia y la microdescompresión vascular constituyen las variantes quirúrgicas más utilizadas en la actualidad por la alta efectividad, sin hablar de coste-efectividad, lo cual es muy difícil, teniendo en cuenta que las investigaciones relacionadas con el tema no abarcan muchas veces el concepto económico. Muchos dan preferencia a la MDV por la posibilidad de utilizarse en pacientes jóvenes evitando las alteraciones sensitivas que provoca la termocoagulación por radiofrecuencia (23-26), sin embargo, en el caso de la termocoagulación por radiofrecuencia se considera más beneficiosa, además, es una técnica bien tolerada por los pacientes ancianos, incluso en los menores de 60 años que la prefieren a pesar de los trastornos sensitivos que originan.

El análisis de evaluación económica podría ser útil en lo que se refiere a decidir cuál de las técnicas quirúrgicas se puede ofrecer y para qué indicaciones resulta ser más efectivo y eficiente. El presente estudio va en esta dirección y representa una base importante para el tratamiento de la NT. Las evaluaciones económicas son acometidas para dar soporte al proceso de toma de decisiones relacionada con la salud, informando a las personas que toman las decisiones

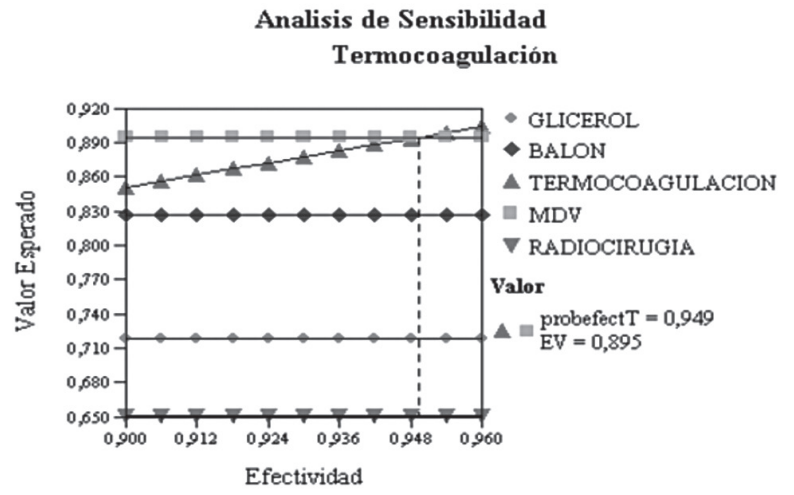

Fig. 3. Análisis univariado de sensibilidad para la termocoagulación. 
TABLA III. ANÁLISIS DE COSTE-EFECTIVIDAD

\begin{tabular}{|c|c|c|c|c|c|c|}
\hline Estrategias & Coste $\$$ & $\begin{array}{l}\text { Coste } \\
\text { marginal }\end{array}$ & Efectividad \% & $\begin{array}{l}\text { Efectividad } \\
\text { marginal }\end{array}$ & $C / E \$ / \%$ & C/E marginal \\
\hline \multicolumn{7}{|l|}{ Etapa 1} \\
\hline Balón & 76,5 & & 0,826 & & 92,52 & \\
\hline Termo coagulación & 86,7 & 10.3 & 0,886 & 0,060 & 7,87 & 171,58 \\
\hline Glicerol & 90,4 & 3.7 & 0,718 & $(0,168)$ & 125,88 & (Domin) \\
\hline Radio cirugía & 228,0 & 141.3 & 0,651 & $(0,235)$ & 350,11 & (Domin) \\
\hline MDV & 391,4 & 304.6 & 0,895 & 0,008 & 437,51 & $36.846,88$ \\
\hline \multicolumn{7}{|c|}{ Etapa 2 (se excluyen las estrategias que fueron dominadas) } \\
\hline Balón & 76,5 & & 0,826 & & 92,52 & \\
\hline Termo coagulación & 86,7 & 10,3 & 0,886 & 0,060 & 7,87 & 171,58 \\
\hline MDV & 391,4 & 304.6 & 0,895 & 0,008 & 437,51 & $36.846,88$ \\
\hline
\end{tabular}

en lo que se refiere a estimar los costes y beneficios de las tecnologías empleadas y su comparación con la práctica clínica prevaleciente $(15,27)$.

En la NT, cuando las metas del tratamiento son la eliminación del dolor facial sin medicamentos y la preservación de la función trigeminal, la cirugía MDV se considera todavía la primera línea de tratamiento, no obstante, la termocoagulación podría representar una opción factible como tratamiento quirúrgico, debida a que su efectividad ya ha sido solidamente investigada (28-33) siendo menos costosa que la MDV, como se reporta en nuestros resultados.

Pollock y Ecker (34), en su estudio de coste-efectividad, dan soporte a los procedimientos percutáneos como más eficientes, debido al bajo coste de estas técnicas quirúrgicas. Aunque en la literatura revisada (35-48) no encontramos información disponible referente a la evaluación de los costes para la termocoagulación, los resultados publicados reflejan que la cirugía menos invasiva atrae a muchos pacientes, lo cual puede ser un factor de peso en cualquier debate entre las diversas opciones quirúrgicas $(15,27,28)$.

Este estudio tiene algunas limitaciones, dado que el análisis de coste no incluye las pérdidas asociadas con la productividad de los pacientes durante el tiempo de ingreso y recuperación, pero si estas hubiesen sido evaluadas, entonces la diferencia entre los dos tratamientos habría resultado aún más grande a favor de la termocoagulación, siendo esta, un servicio ambulatorio para el paciente.

\section{CONCLUSIONES}

Considerando que la disyuntiva actual en el tratamiento de la NT se basa en los que patrocinan la microdescompresión vascular por su efectividad y pocos trastornos sensitivos, y por otro lado están los que defienden a la termocoagulación por su efectividad y beneficios. Concluimos que

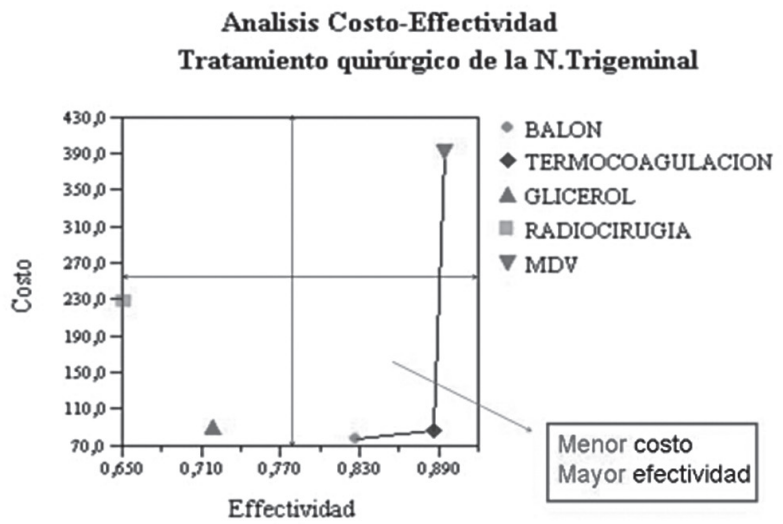

Fig. 4. Análisis de coste-efectividad para el tratamiento quirúrgico de la neuralgia trigeminal.

la alternativa de la termocoagulación por radiofrecuencia es más beneficiosa, ofrece escasas complicaciones y es menos costosa.

CORRESPONDENCIA:

Dra. Isabel Mora Díaz

Departamento de Investigaciones y Proyectos

Hospital Clínico Quirúrgico “Hermanos Ameijeiras”

La Habana, Cuba

Teléfono: 078700937

Correo electrónico: juriag@infomed.sld.cu

\section{BIBLIOGRAFÍA}

1. Tatli M, Satici O, Kanpolat Y, et al. Various surgical modalities for trigeminal neuralgia:literature study of respective long-term outcomes. Acta Neurochir. 2008;150:243-55. 
2. Robaina F. Neuralgia del Trigémino. Revisión del tratamiento médico y quirúrgico. Rev Soc Esp Dolor. 2008;4:248-56.

3. Lopez BC, Peter JH, Zakrzewska JM. Systematic review of ablative neurosurgical techniques for the treatment of trigeminal neuralgia. Neurosurgery. 2004;54:973-83.

4. Haridas A, Mathewson C, Eljamel S. Long-term results of 405 refractory trigeminal neuralgia surgeries in 256 patients. Zentralbl Neurochir. 2008 Nov;69(4):170-4.

5. Spatz AL, Zakrzewska JM, Kay EJ. Decision analysis of medical and surgical treatments for trigeminal neuralgia: How patient evaluations of bene.ts and risks a.ect the utility of treatment decisions. Pain. 2007;131:302-10.

6. Brisman R. Microvascular decompression vs. gamma knife radiosurgery for typical trigeminal neuralgia: preliminary findings. Stereotact Funct Neurosurg. 2007;85:94-8.

7. Kondo A. Microvascular decompression surgery for trigeminal neuralgia. Stereotact Funct Neurosurg. 2001;77:187-9.

8. Olson S, Atkinson L, Weidmann M. Microvascular decompression for trigeminal neuralgia: recurrences and complications. J Clin Neurosci. 2005;12:787-9.

9. Sindou M, Leston J, Howeidy T, et al. Micro-vascular decompression for primary Trigeminal Neuralgia (typical or atypical). Long-term effectiveness on pain; prospective study with survival analysis in a consecutive series of 362 patients. Acta Neurochir (Wien). 2006;148:1235-45.

10. Fountas KN, Lee GP, Smith JR. Outcome of patients undergoing gamma knife stereotactic radiosurgery for medically refractory idiopathic trigeminal neuralgia: medical college of Georgia's experience. Stereotact Funct Neurosurg. 2006;84:88-96.

11. Kondziolka D, Lacomis D, Niranjan A, et al. Histological effects of trigeminal nerve radiosurgery in a primate model:implications for trigeminal neuralgia radiosurgery. Neurosurgery. 2000;46:971-6.

12. Pollock BE, Phuong LK, Gorman DA, et al. Stereotactic radiosurgery for idiopathic trigeminal neuralgia. J Neurosurg. 2002;97:347-53.

13. Pollock BE. Radiosurgery for trigeminal neuralgia: is sensory disturbance required for pain relief? J Neurosurg. 2006;105(Suppl):103-6.

14. Sánchez Pedraza R, Echeverry Raad J. Análisis de decisiones clínicas: estrategia práctica para la selección de la mejor alternativa en el manejo de pacientes. Rev. Colombiana de Pediatría. 2004, Volumen 39 (3).

15. Tarricone R, Aguzzi G, Musi F, et al. Cost-effectiveness analysis for trigeminal neuralgia: Cyberknife vs microvascular decompression. Neuropsychiatr Dis Treat. 2008 June;4(3):647-52.

16. Petitti DB. Meta-Analysis, decision analysis and cost-effectiveness analysis. Methods for Quantitative Synthesis in Medicine. New York: Oxford University Press: 2000;17.

17. Análisis de costo-utilidad de la perfusión miocárdica en reposo y las troponinas en el tamizaje de pacientes con dolor torácico y electrocardiograma normal o no diagnóstico en urgencias. Year 7, Number 26, October 2004. Article $\mathrm{N}^{\circ}$ AJ26-2.

18. Mittal B, Thomas DG. Controlled thermocoagulation in trigeminal neuralgia. J Neurology. 1986;49:932.

19. Taha JM, Tew JM. Comparison of surgical treatments for trigeminal neuralgia: reevaluation of radiofrequency rhizotomy. Neurosurgery. 1997 May;40(5):1106-7.

20. Ashkan K, Marsh H. Microvascular decompression for trigeminalneuralgia in the elderly: a review of the safety and e cacy. Neurosurgery. 2004;55:840-8.
21. Barker FG, Jannetta PJ, Bissonette DJ, et al. Thelong-term outcome of microvascular decompression for trigeminalneuralgia. N Engl J Med. 1996;334:1077-83.

22. Barker FG, Jannetta PJ, Bissonette DJ, et al. Trigeminal numbnessand tic relief after microvascular decompression for typicaltrigeminal neuralgia. Neurosurgery. 1997;40:39-45.

23. Martinez JE, et al Estudio comparativo entre la termocoagulación gasseriana y la microcompresión por balón catéter. Rev Esp Dolor. 2005;12(1):3-7.

24. Kao MC. Re Validity of percutaneous controlled radiofrequency thermocoagulation in the treatment of isolated third division trigeminal neuralgia. Surg Neurol. 2009;72(1):99100.

25. Salama H, Ben-Khayal H, Abdel SM, et al. Outcome of medical and surgical management in intractable idiopathic trigeminal neuralgia. Ann Indian Acad Neurol. 2009 JulSep;12(3):173-8.

26 Bederson JB, Wilson CB. Evaluation of microvascular decompression and partial sensory rhizotomy in 252 cases of trigeminal neuralgia. J Neurosurg. 1989;71:359-67.

27. Concepción Pérez MT, Saldaña AN, Martínez S, et al. Trigeminal neuralgia treated with pregabalin in Family Medicine Settings: It's Effect on Pain alleviation and Cost Reduction. J Clin Pharmacol. 2009;49:582.

28. Zakrzewska JM, Lopez MD, Benjamin C. Quality of reporting in evaluations of surgical treatment of trigeminal neuralgia: recommendations for future reports. Neurosurgery. 2003 July;53(1):110-22.

29. Scott WJ, Benedict W, Owen K, et al. Perioperative and longterm operative outcomes after surgery for trigeminal neuralgia: microvascular decompression vs percutaneous balloon ablation. Head Face Med. 2008;4:11.

30. Pringle M. Surgical treatment for trigeminal neuralgia. BMJ 1996 Oct.;313.

31. Adams CB, Kaye AH, Teddy PJ. The treatment of trigeminal neuralgia by posterior fossa microsurgery. J. Neurol Neurosurg Psychiatry. 1982;45:1020-6.

32. Broggi G, Ferroli P, Franzini A, et al. Microvascular decompression for trigeminal neuralgia: comments on a series of 250 cases. J Neurol Neurosurg Psychiatry. 2000;68:59-64.

33. Silverberg GD, Britt RH. Percutaneous radio-frequency rhizotomy in the treatment of trigeminal neuralgia. J Med. 1978;129:97-100.

34. Pollock BE, Ecker RD. A Prospective Cost-Effectiveness Study of Trigeminal Neuralgia Surgery. Clin J Pain. 2005;21:317-22.

35. Ong KS, Keng SB. Evaluation of Surgical Procedures for Trigeminal Neuralgia. Anesthesiology. 2003;50:181-8.

36. Lopez BC, Hamlyn PJ, Zakrzewska JM. Stereotactic radiosurgery for primary trigeminal neuralgia: state of the evidence and recommendations for future reports. J Neurol Neurosurg Psychiatry. 2004;75:1019-24.

37. Merrison AF, Fuller G. Treatment options for trigeminal neuralgia. BMJ 2003;327:1360-1.

38. Oh IH, Choi SK, Park BJ, et al. The Treatment Outcome of Elderly Patients with Idiopathic Trigeminal Neuralgia: Micro-Vascular Decompression versus Gamma Knife Radiosurgery. J Korean Neurosurgery Soc. 2008;44:199-204.

39. Trigeminal neuralgia: treat but do not prolong. Br Med J. 1981;282:1410.

40. Sengupta RP, Stunden RJ. Radiofrequency thermocoagulation of Gasserian ganglion and its rootlets for trigeminal neuralgia. Br Med J. 1977;1:142-3. 
41. Lobato RD, Rivas JJ, Sarabia R, et al. Percutaneous microcompressionof the gasserian ganglion for trigeminal neuralgia. J Neurosurg. 1990;72:546-53.

42. MacDonald BK, Cockerell OC, Sander JW, et al. Theincidence and lifetime prevalence of neurological disorders in aprospective community-based study in the UK. Brain. 2000;123:665-76.

43. Mathews ES, Scrivani SJ. Percutaneous stereotactic radiofrequencythermal rhizotomy for the treatment of trigeminal neuralgia. J Med. 2000;67:288-99.

44. Nurmikko TJ, Eldridge PR. Trigeminal neuralgia-pathophysiology, diagnosis and current treatment. Br J Anaesth. 2001;87:117-32.
45. Yoon KB, Wiles JR, Miles JB, et al. Long-term outcome ofpercutaneous thermocoagulation for trigeminal neuralgia. Anaesthesia. 1999;54:803-8.

46. Young RF. Glycerol rhizolysis for treatment of trigeminal neuralgia. J Neurosurg. 1988;69:39-45.

47. Zakrzewska JM. Trigeminal neuralgia. In: Zakrzewska JM, Harrison SD, (editors). Assessment and management of orofacialpain. Amsterdam: Elsevier; 2002. p. 267-370.

48. Zakrzewska JM, Lopez BC. Trigeminal neuralgia: Clinical Evidence, 2005;14:426-8. Disponible en: http/ /www.clinicalevidence.com. Citado 24 Octubre 2010. 